Document downloaded from:

http://hdl.handle.net/10251/64841

This paper must be cited as:

Ballester Bolinches, A.; Esteban Romero, R.; Jiang, Q.; Li, X. (2015). On a class of generalised Schmidt groups. Annali di Matematica Pura ed Applicata. 194(1):77-86. doi:10.1007/s10231-013-0365-3.

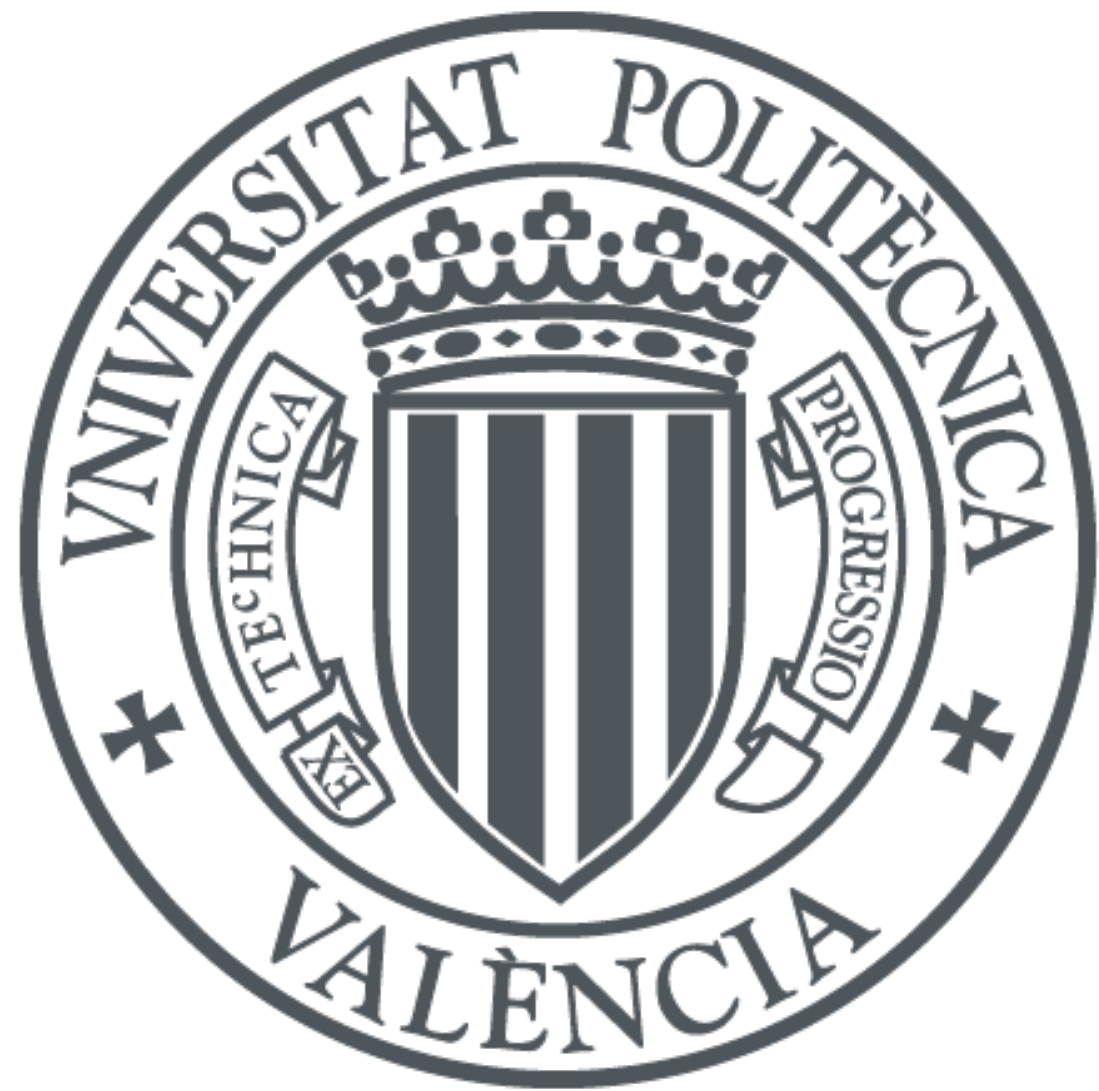

The final publication is available at

http://link.springer.com/article/10.1007\%2Fs10231-013-0365-3

Copyright Springer; Fondazione Annali di Matematica Pura ed Applicata

Additional Information

The final publication is available at Springer via http://dx.doi.org/10.1007/s10231-013-03653 


\title{
On a class of generalised Schmidt groups
}

\author{
A. Ballester-Bolinches* \\ Qinhui Jiang
}

\author{
R. Esteban-Romero ${ }^{\dagger}$ \\ Xianhua $\mathrm{Li}^{\S}$
}

\begin{abstract}
In this paper families of non-nilpotent subgroups covering the nonnilpotent part of a finite group are considered. An $A_{5}$-free group possessing one of these families is soluble, and soluble groups with this property have Fitting length at most three. A bound on the number of primes dividing the order of the group is also obtained.

Mathematics Subject Classification (2010): 20D05, 20D10, 20F16,

Keywords: finite groups, nilpotent groups, maximal subgroups.
\end{abstract}

\section{Introduction and statement of results}

All groups considered in this paper are finite.

The results presented here spring from the classical results of Schmidt [13] about the structure of the minimal non-nilpotent groups and later developments from them ([12], [2], [3], [9], [10]). Schmidt proved that if all the maximal subgroups of a group $G$ are nilpotent, then $G$ is soluble, and that, in addition, if $G$ is not nilpotent, $|G|$ has exactly two distinct prime factors, $G$ has a normal Sylow subgroup and a cyclic non-normal Sylow subgroup. These groups are called minimal non-nilpotent groups or Schmidt groups.

Rose [12] studied the effects of replacing maximal subgroups by nonnormal (or abnormal) maximal subgroups in the hypothesis of Schmidt's result, and the following fact is established:

*Departament d'Àlgebra, Universitat de València; Dr. Moliner, 50; 46100, Burjassot, València, Spain; email: Adolfo.Ballester@uv.es

${ }^{\dagger}$ Institut Universitari de Matemàtica Pura i Aplicada, Universitat Politècnica de València; Camí de Vera, s/n; 46022 València, Spain, email: resteban@mat.upv.es

${ }^{\ddagger}$ Department of Mathematics, Shanghai University, 200444, Shanghai, School of Mathematical Sciences, University of Jinan, 250002 Jinan, China; email: syjqh20010163.com

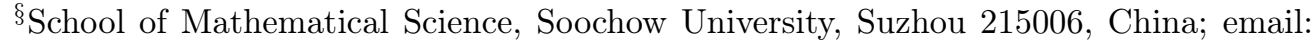
xhli@suda.edu.cn 
Theorem A. If every non-normal maximal subgroup of a group $G$ is nilpotent, then $G$ has a normal Sylow subgroup $P$ such that $G / P$ is nilpotent.

We shall say that a group $G$ is a Rose group if every non-normal maximal subgroup of $G$ is nilpotent.

In a recent paper [11], Li and Guo characterised Rose groups by means of certain families of normal non-nilpotent subgroups, and obtained more detailed information about the number of primes dividing the order of the group. They also gave an alternative proof for solubility.

Theorem B. Let $G$ be a Rose group. Then

1. $G$ is soluble;

2. $G$ is p-nilpotent for some prime $p$;

3. If $G$ is non-nilpotent, then $2 \leq|\pi(G)| \leq k+2$, where $k$ is the number of normal maximal subgroups of $G$ which are not nilpotent.

The present paper furnishes extensions of the main results of Rose, Li and Guo, and was motivated by some ideas of the paper [11]. We consider families of non-nilpotent subgroups covering the non-nilpotent part of the group, and analyse how they determine the group structure.

It is abundantly clear that our results are not a mere exercise in generalisation. In fact, Theorem A and Theorem B cannot be extended directly: the alternating group of degree 5 is a fundamental obstruction to get solubility. We must seek to discover how nearly a non-nilpotent group with some of our coverings is soluble. With this purpose in view, we consider the solubility question (Theorem $\mathrm{C}$ ), and give more detailed structural information in the soluble case.

Definition 1.1. Let $G$ be a non-nilpotent group. A Schmidt covering of $G$ is a, possibly empty, family of non-nilpotent proper subgroups $\left\{K_{1}, \ldots, K_{n}\right\}$ of $G$ satisfying the following two conditions:

1. If $i, j \in\{1, \ldots, n\}$ and $i \neq j$, then $K_{i}$ is not contained in $K_{j}$.

2. If $T$ is a proper subgroup of $G$ such that $T \notin\left\{K_{1}, \ldots, K_{n}\right\}$ and $T$ is not contained in $K_{t}$ for some $t \in\{1, \ldots, n\}$, then $T$ is nilpotent provided that $T$ is a supplement in $G$ of the nilpotent residual $G^{\mathfrak{N}}$ of $G$.

Recall that the nilpotent residual of a group is the smallest normal subgroup with nilpotent quotient. We say that a group $G$ is an $N N C$-group if $G$ has a Schmidt covering. If $G$ is a Schmidt group, then $G$ is an $N N C$-group 
with an empty Schmidt covering, and if $G$ is a non-nilpotent Rose group, then the empty set and the set of all non-nilpotent normal maximal subgroups of $G$ are both Schmidt coverings of $G$. Hence $G$ is an $N N C$-group. However, the symmetric group of degree 4 shows that the class of Rose groups is a proper subclass of the class of all $N N C$-groups.

Our first main theorem is the following.

Theorem C. Let $G$ be an $N N C$-group. If $G$ has no section isomorphic to $A_{5}$, then $G$ is soluble.

We note that the proof of the above result relays on the Classification of Finite Simple Groups.

For $G$ a nontrivial soluble group, we let $F(G)$ denote the Fitting subgroup of $G$. The subgroups $F_{i}(G)$ are defined inductively by $F_{0}(G)=1$, and $F_{i+1}(G) / F_{i}(G)=F\left(G / F_{i}(G)\right)$. The smallest non-negative integer $n$ such that $F_{n}(G)=G$ is the Fitting length $l(G)$ of $G$. The trivial group has Fitting length 0; a nontrivial nilpotent group has Fitting length 1; and if $G \neq 1$, then $l(G / \mathrm{F}(G))=l(G)-1$.

According to Theorem A, a Rose group has Fitting length at most 2. The symmetric group of degree 4 is an $N N C$-group of Fitting length 3 . Hence, Theorem A does not hold for soluble $N N C$-groups. However, we have:

Theorem D. Let $G$ be a soluble $N N C$-group. Then the Fitting length of $G$ is at most 3 .

In view of the third assertion of Theorem $\mathrm{B}$, it is of interest to inquire whether there is a bound on the number of distinct prime factors of $|G|$, at least when $G$ is a soluble $N N C$-group. Note that every non-empty Schmidt covering contains every conjugacy class of abnormal maximal subgroups, and a bound for the number of distinct primes dividing the order of a group is naturally related to the number of conjugacy classes of subgroups contained in the maximal covering.

Let $G$ be an $N N C$-group. We say that the Schmidt covering $\mathscr{A}$ is the maximal covering of $G$ if $\mathscr{A}$ contains every non-nilpotent maximal subgroup of $G$.

Theorem E. Let $G$ be a soluble $N N C$-group. Let $\mathscr{A}$ be the maximal covering of $G$. Then $2 \leq|\pi(G)| \leq l+2$, where $l$ is the number of conjugacy classes of subgroups contained in $\mathscr{A}$.

Note that the order of a Schmidt group is divisible by two different primes, and if $A$ is a Schmidt group and $p$ is a prime which does not divide its order, then $G=A \times B$, where $B$ is a cyclic group of order $p$, is an $N N C$-group and $\{A\}$ is the maximal Schmidt covering of $G$. Hence the bounds of the above theorem are best possible. 


\section{Preliminaries}

Before taking up the proofs of our main results, we shall give in this section a few very useful results on $N N C$-groups.

Recall that a subgroup $H$ of a group $G$ is abnormal in $G$ if $g \in<H, H^{g}>$ for all $g \in G$. Our first result shows that every abnormal subgroup in a Schmidt covering of $G$ should be a maximal subgroup of $G$.

Proposition 2.1. Let $\left\{K_{1}, \ldots, K_{n}\right\}$ be a Schmidt covering of an NNC-group $G$. If, for some $j \in\{1, \ldots, n\}, K_{j}$ is not maximal in $G$, then there exists a normal maximal subgroup $L$ of $G$ containing $K_{j}$ such that $\{L\} \bigcup\left\{K_{t}: K_{t} \not \leq\right.$ $L\}$ is a Schmidt covering of $G$.

Proof. Suppose that $K_{j}$ is not maximal in $G$, for some $j \in\{1, \ldots, n\}$. Let $L$ be a maximal subgroup of $G$ containing $K_{j}$. Clearly, $L \notin\left\{K_{1}, \ldots, K_{n}\right\}$ and $L \not K_{j}$. If $L$ were not normal in $G$, we would have $G=G^{\mathfrak{N}} L$; and since $\left\{K_{1}, \ldots, K_{n}\right\}$ is a Schmidt covering of $G$, this would imply the nilpotency of $L$. Hence $L$ is normal in $G$. Clearly $\{L\} \cup\left\{K_{t}: K_{t} \not \leq L\right\}$ is a Schmidt covering of $G$.

Corollary 2.2. Let $G$ be an $N N C$-group which is not a Schmidt group. Then $G$ has a Schmidt covering composed of maximal subgroups of $G$, and every Schmidt covering of $G$ contains each non-nilpotent abnormal maximal subgroup of $G$.

As we said in the introduction, the symmetric group of degree 4 is a typical example of an $N N C$-group which is not a Rose group. However, $N N C$-groups with small Schmidt coverings are Rose groups.

Proposition 2.3. Let $\mathscr{A}=\left\{K_{1}, \ldots, K_{n}\right\}$ be a Schmidt covering of an NNCgroup $G$. If the number of maximal subgroups of $G$ in $\mathscr{A}$ is at most 2 , then $G$ is a Rose group.

Proof. We may suppose that $\mathscr{A}$ is non-empty. Let $k$ denote the number of maximal subgroups of $G$ in $\mathscr{A}$.

Assume $k=1$, and $K_{1}$ is the unique maximal subgroup of $G$ in $\mathscr{A}$. Let $L \neq K_{1}$ be a maximal subgroup of $G$ conjugate to $K_{1}$ in $G$. Since $L$ is not nilpotent, it follows that $L$ belongs to $\mathscr{A}$. Hence $K_{1}$ is normal in $G$ and then every abnormal maximal subgroup of $G$ is nilpotent. Then $G$ is a Rose group.

Suppose $k=2$, and that $K_{1}$ is one of the maximal subgroups of $G$ in $\mathscr{A}$. If $K_{1}$ were not normal in $G$, then $\mathscr{A}$ would contain any conjugate of $K_{1}$ in $G$. This would mean that $\left|G: K_{1}\right|=2$, and $K_{1} \unlhd G$, contrary to our assumption. Hence the maximal subgroups of $G$ in $\mathscr{A}$ are normal in $G$, and $G$ is a Rose group. 
Lemma 2.4. Let $G$ be an $N N C$-group with a Schmidt covering $\left\{K_{1}, \ldots, K_{n}\right\}$. Assume that, for some $i \in\{1, \ldots, n\}, K_{i}$ is not contained in any normal maximal subgroup of $G$. Then $n>2$, and $K_{i}$ is a Rose group.

Proof. According to Proposition 2.1, $K_{i}$ is an abnormal maximal subgroup of $G$. Hence, $G=G^{\mathfrak{N}} K_{i}$. Since $K_{i}$ is not nilpotent, it follows that $n>2$. Let $T$ be a maximal subgroup of $K_{i}$ such that $K_{i}=K_{i}^{\mathfrak{N}} T$. Then $G=G^{\mathfrak{N}} T$. Since $T \notin\left\{K_{1}, \ldots, K_{n}\right\}$, we conclude that that $T$ is nilpotent. Consequently, $K_{i}$ is a Rose group,

The next result is particularly useful when an inductive argument involving quotient groups is applied.

Lemma 2.5. Let $G$ be an $N N C$-group with a Schmidt covering $\mathscr{S}=\left\{K_{1}, \ldots, K_{n}\right\}$. If $N$ is a normal subgroup of $G$, then one of the following statements holds:

1. $G / N$ is nilpotent;

2. $G=N K_{i}$ for all $i \in\{1, \ldots, n\}$ and $G / N$ is a Rose group;

3. $\left\{K_{i} / N: N \leq K_{i}, K_{i} / N\right.$ is non-nilpotent $\}$ is a Schmidt covering of $G / N$.

In other words, $G / N$ is either nilpotent or an $N N C$-group.

Proof. Suppose that $G$ is a Schmidt group. If $N$ is contained in the Frattini subgroup of $G$, it follows that $G / N$ is a Schmidt group, and if $N$ is supplemented in $G$ by a maximal subgroup of $G$, then $G / N$ is nilpotent. Hence lemma holds in this case. Therefore, by Corollary 2.2, we may assume that $\mathscr{S}$ is a non-empty Schmidt covering of $G$ composed of maximal subgroups. Suppose, now, that $G / N$ is not nilpotent. If $N$ is not contained in any $K_{i}$, then $G=N K_{i}$ for all $i \in\{1, \ldots, n\}$. Let $T / N$ be an arbitrary maximal subgroup of $G / N$. Then $T \notin\left\{K_{1}, \ldots, K_{n}\right\}$, and so either $T \unlhd G$ or $T$ is nilpotent. Consequently, $G / N$ is a Rose group and Statement 2 holds.

Assume that $\mathscr{A}:=\left\{K_{1}, \ldots, K_{r}\right\}$ is the non-empty set of subgroups of $\left\{K_{1}, \ldots, K_{n}\right\}$ containing $N, 1 \leq r \leq n$, and write $\mathscr{C}:=\left\{K_{i} / N\right.$ : $K_{i} / N$ is non-nilpotent, $\left.1 \leq i \leq r\right\}$. We show that $\mathscr{C}$ is a Schmidt covering of $G / N$. If $\mathscr{C}$ is empty, then every abnormal maximal subgroup of $G$ containing $N$ is nilpotent. Hence $G / N$ is a Rose group and lemma holds in this case. Suppose that $\mathscr{C}$ is non-empty. It is clear that $\mathscr{C}$ satisfies Condition 1 of Definition 1.1. Let $T / N$ be a proper subgroup of $G / N$ such that $T / N \notin \mathscr{C}$, $T / N \not K_{t} / N \in \mathscr{C}$, and $G / N=(G / N)^{\mathfrak{N}} \cdot T / N$. Then $G=G^{\mathfrak{N}} T$. If $T \leq K_{j}$ for some $1 \leq j \leq r$ such that $K_{j} / N$ is nilpotent, then $T / N$ is also nilpotent. Otherwise, $T \notin\left\{K_{1}, \ldots, K_{n}\right\}$. Since $\mathscr{S}$ is a Schmidt covering of $G, T$ is 
nilpotent. In both cases, we conclude that $T / N$ is nilpotent. Therefore $\mathscr{C}$ is a Schmidt covering of $G / N$ and Statement 3 holds.

Proposition 2.6. Let $\mathscr{A}=\left\{K_{1}, \ldots, K_{n}\right\}$ be a family of non-nilpotent subgroups of a group $G$ such that $K_{i}$ is not contained in $K_{j}$, if $i \neq j, i, j \in$ $\{1, \ldots, n\}$. Then $\mathscr{A}$ is a Schmidt covering of $G$ if and only if every proper supplement of the nilpotent residual of $G$ not belonging to $\mathscr{A}$ is nilpotent.

Proof. It is clear that only the necessity of the condition is in doubt. Assume that $\mathscr{A}$ is a Schmidt covering of $G$ and let $1 \neq T$ be a proper subgroup of $G$ such that $G=G^{\mathfrak{N}} T$ and $T$ does not belong to $\mathscr{A}$. We prove that $T$ is nilpotent by induction on the order of $G$. If $T$ is not contained in some $K_{i}$, then $T$ is nilpotent. Hence we may assume that $T \leq \cap\left\{K_{i}: 1 \leq i \leq n\right\}$ and $1 \neq G^{\mathfrak{N}}$. In particular, $\mathscr{A}$ is non-empty and so $\mathscr{A}$ contains every abnormal maximal subgroup of $G$. Let $X$ be the intersection of the abnormal maximal subgroups of $G$. Then, $X$ is normal in $G$ and, if $G$ is non-soluble, $X$ is nilpotent by [14]. Hence $T$ is nilpotent. Assume that $G$ is soluble. Since $X$ is a proper normal subgroup of $G$, there exists a maximal normal subgroup $Y$ of $G$ containing $X$. Then $G / Y$ is nilpotent, and so $G^{\mathfrak{N}}$ is contained in $Y$. Therefore $G=Y$, contradicting the maximality of $Y$.

\section{Proofs of the main results}

Proof of Theorem C. Suppose that the result is false, and let the group $G$ provide a counterexample of least possible order. Then, by Proposition 2.3 and Theorem A, the number of abnormal maximal subgroups in every Schmidt covering of $G$ is greater than 2. Since the properties of $G$, as enunciated in the statement of the theorem, are inherited by non-nilpotent quotients of $G$, the minimality of $G$ implies that $G$ has a unique minimal normal subgroup, say $N . N$ must be insoluble, $C_{G}(N)=1$, and $N$ is a direct power of a simple non-abelian group. Assume that $G / N$ is not nilpotent, and let $H / N$ be a non-normal maximal subgroup of $G / N$. Then $H$ is a nonnormal maximal subgroup of $G$ supplementing the nilpotent residual of $G$. If $H$ is not in any Schmidt covering of $G$, then $H$ is nilpotent; otherwise, $H$ is a Rose group by Lemma 2.4. In both cases, $H$ should be soluble, contrary to assumption. Therefore $G / N$ is nilpotent and $N=G^{\mathfrak{N}}$ is the nilpotent residual of $G$.

Let $\mathscr{A}$ be a Schmidt covering of $G$ composed of maximal subgroups. Write $N=S_{1} \times \cdots \times S_{m}$, where $S_{j}$ are pairwise isomorphic non-abelian simple groups, $j \in\{1, \ldots, m\}$. Let us denote $C=\mathrm{C}_{G}\left(S_{1}\right), M=\mathrm{N}_{G}\left(S_{1}\right)$, 
and $K=S_{2} \times \cdots \times S_{m}$. Let $H$ be a core-free maximal subgroup of $G$. Then $H$ is soluble. Write $V=M \cap H$.

Applying $[1,1.1 .52]$ one of the following statements holds:

1. $G$ is an almost simple group;

2. $(G, H)$ is equivalent to a primitive pair with simple diagonal action; in this case, $H \cap N$ is a full diagonal subgroup of $N$;

3. $(G, H)$ is equivalent to a primitive pair with product action such that $H \cap N \cong D_{1} \times \cdots \times D_{l}$, a direct product product of $l>1$ subgroups such that, for each $j=1, \ldots, l$, the subgroup $D_{j}$ is a full diagonal subgroup of a direct product $\prod_{i \in \mathcal{I}_{j}} S_{i}$.

4. $(G, H)$ is equivalent to a primitive pair with product action such that the projection $R_{1}=(H \cap N)^{\pi_{1}}$ is a non-trivial proper subgroup of $S_{1}$; in this case $R_{1}=V C \cap S_{1}$ and $V C / C$ is a maximal subgroup of the almost simple group $M / C$, where $\pi_{1}$ is a projection from $N$ to $S_{1}$;

5. $H \cap N=1$.

The solubility of $H$ implies that $H \cap N$ cannot contain any copy of the composition factor of $N$. If $H \cap N=1$, we know, by a result of Lafuente (see $[1,1.1 .51(2)]$ ), that $H$ would be a primitive group with non-abelian socle. Since $H$ is soluble, it follows that $G$ is an almost simple group or $(G, H)$ is equivalent to a primitive pair with product action such that the projection $R_{1}=(H \cap N)^{\pi_{1}}$ is a non-trivial proper subgroup of $S_{1}$. Assume that $N$ is not simple. Since $M / C$ is almost simple, we have that $M / K$ is neither nilpotent nor a Rose group. In addition, $N / K$ is the nilpotent residual of $M / K$. Let $L / K$ a non-nilpotent maximal subgroup of $M / K$ supplementing $N / K$ in $M / K$. Applying [1, 1.1.35], there exists a maximal subgroup $A$ of $G=A N$ such that $L=(A \cap M) K$. Then $A$ belongs to $\mathscr{A}$. Since every supplement $U / K$ of $N / K$ in $M / K$ is of the form $U / K=$ $(B \cap M) K / K$ for some supplement $B$ of $N$ in $G$ by [1,1.1.35], we conclude that the set of all non-nilpotent maximal subgroups of $M / K$ supplementing $N / K$ is a Schmidt covering of $M / K$. Therefore $M / K$ is an $N N C$-group with no sections isomorphic to $A_{5}$. The minimal choice of $G$ implies that $M / K$ is soluble, contradicting the fact that $N / K$ is non-abelian simple. Thus $G$ must be an almost simple group.

Let $p \geq 5$ be a prime dividing $|N|$, and let $P$ be a Sylow $p$-subgroup of $N$. Then $G=N \mathrm{~N}_{G}(P)=G^{\mathfrak{N}} \mathrm{N}_{G}(P)$, and so $\mathrm{N}_{G}(P)$ is either contained in some maximal abnormal subgroup of $\mathscr{A}$ or $\mathrm{N}_{G}(P)$ is nilpotent. If the latter were true, then we would have $\mathrm{O}^{p}(N)<N$ by [6, X, 8.13], contrary to 
hypothesis. Therefore $Z=\mathrm{N}_{G}(P)$ is contained in some abnormal maximal subgroup $E$ belonging to $\mathscr{A}$. By Lemma 2.4, $E$ is a Rose group. Applying Theorem A, $Z^{\mathfrak{N}}$ is a $q$-group for some prime $q$. Suppose that $q \neq p$, and let $Q$ be a Sylow $q$-subgroup of $Z$. Then $Q$ is normal in $Z$ and $Z=Q Z_{q^{\prime}}$, where $Z_{q^{\prime}}$ is a Hall $q^{\prime}$-subgroup of $Z$. In particular, $Z_{q^{\prime}}$ is nilpotent. Moreover, $Z_{q^{\prime}}$ cannot be a normal subgroup of $Z$, otherwise, we should have $Z$ nilpotent, contradicting our hypothesis. Hence $\mathrm{N}_{Z}\left(Z_{q^{\prime}}\right)$ is contained in a non-normal maximal subgroup $Y$ of $Z$. Let $W$ be a maximal subgroup of $E$ containing $Y$. Then $W$ is not normal in $E$ and so $W$ is nilpotent. Thus $Y$ is nilpotent and $Z_{q^{\prime}}$ centralises $P$. Since $Q$ also centralises $P$, we have that $Z / \mathrm{C}_{G}(P)$ is a $p$-group. Applying [6, X, 8.13], $\mathrm{O}^{p}(N)<N$, contradicting the prescribed minimality of $N$. Consequently $q=p$. Then the nilpotent residual of $E$ is a $p$-group and so a Sylow $p$-subgroup of $E$ is normal in $E$. It implies that $E=Z$. In this case, $P$ is a Sylow $p$-subgroup of $G$.

Assume that $G$ is not simple. Applying [8, 1.1], there exists a normal subgroup $G_{0}$ of $G$ which is minimal such that $E_{0}:=E \cap G_{0}$ is maximal in $G_{0}$, and $E$ is isomorphic to $E_{0} \cdot G / G_{0}$. Moreover, with the exceptions for which the numbers $c$ of conjugacy classes of such subgroups $E_{0}$ are listed in [8, Table 1], all of such subgroups $E_{0}$ are conjugate. Note that the subgroups $E_{0}$ contain a Sylow subgroup of $G$ for a prime $p \geq 5$. Therefore $N$ cannot be isomorphic to any simple group in [8, Table 1]. Hence all of such subgroups $E_{0}$ are conjugate and they are the normalisers of a Sylow $p$-subgroup of $G$ for fixed prime $p \geq 5$ dividing the order of $N$. This implies that $|N|$ is divisible by exactly three different primes. According to [7, Table 1], $N$ is isomorphic to $A_{5}, A_{6}$, $\operatorname{PSU}(4,2), \operatorname{PSL}(2,7), \operatorname{PSL}(2,8), \operatorname{PSU}(3,3), \operatorname{PSL}(3,3)$ or PSL $(2,17)$. Clearly $N$ cannot be isomorphic to $A_{5}, A_{6}$ or $P S U(4,2)$ since $G$ is $A_{5}$-free. Since the nilpotent residual of $G$ is isomorphic to $N, G \cong A u t(N)$ and $G$ is an almost simple group, it follows by Atlas [4] that $|G: N|=2$ if $N \in\{\operatorname{PSL}(2,7)$, $\operatorname{PSU}(3,3), \operatorname{PSL}(3,3), \operatorname{PSL}(2,17)\}$ and $|G: N|=3$ if $N=\operatorname{PSL}(2,8)$. Let $p$ be the largest prime divisor of $|N|$ and $P$ a Sylow $p$-subgroup of $N$. Then $|P|=p$ and $N_{G}(P)$ belongs to $\mathscr{A}$. By [4], $N_{G}(P)$ is isomorphic to $7: 6$ if $N$ is isomorphic to one of the groups $\operatorname{PSL}(2,7), \operatorname{PSL}(2,8)$, or $\operatorname{PSU}(3,3)$; it is isomorphic to $13: 6$ if $N \cong \operatorname{PSL}(3,3)$, or isomorphic to $17: 16$ if $N \cong \operatorname{PSL}(2,17)$. Hence $N_{G}(P)$ has a subgroup $K$ isomorphic to $7: 2,7: 3$, $7: 2,13: 2,17: 8$, if $N$ is isomorphic to $\operatorname{PSL}(2,7), \operatorname{PSL}(2,8), \operatorname{PSU}(3,3)$, $\operatorname{PSL}(3,3)$, or PSL $(2,17)$ respectively. Since $N_{G}(P)$ is a Rose group, we have $K$ is nilpotent. This contradiction implies that $G$ cannot be an almost simple group.

Consequently, $G$ is a simple group. Then every maximal subgroup of $G$ either is nilpotent or belongs to $\mathscr{A}$. Therefore every maximal subgroup is either nilpotent or a Schmidt group. By [5, II, 7.5], $G$ is isomorphic to one 
of the following groups:

1. $\operatorname{PSL}(2, p)$, where $p$ is a prime, $p>3$, and $p^{2}-1 \not \equiv 0(\bmod 5)$;

2. $\operatorname{PSL}\left(2,2^{q}\right)$, where $q$ is a prime,

3. $\operatorname{PSL}\left(2,3^{q}\right)$, where $q$ is an odd prime,

4. $\operatorname{PSL}(3,3)$, or

5. $\mathrm{Sz}\left(2^{q}\right)$, where $q$ is an odd prime.

In the following, we analyse each one of these cases and derive a contradiction.

1. $G \cong \operatorname{PSL}(2, p)$, where $p>3$ is a prime and $p^{2}-1 \not \equiv 0(5)$.

Applying [5, II, 8.27], $G$ has subgroups $G_{1} \cong D_{r}$ and $G_{2} \cong D_{s}$, where $r=2 \cdot \frac{p+1}{(2, p-1)}=p+1$ and $s=2 \cdot \frac{p-1}{(2, p-1)}=p-1$. Since $G_{1}$ and $G_{2}$ are either nilpotent or Schmidt groups, it follows that $\frac{r}{2}=\frac{p+1}{2}$ and $\frac{s}{2}=\frac{p-1}{2}$ are either a power of 2 or a prime, Note that $p-1, p, p-1$ are consecutive integers, and so 3 must divide one of them. As $p>3$ is a prime, we have that $3 \nmid p$. Assume that $3 \mid p+1$. Then $6 \mid p+1$, which implies $3 \mid \frac{p+1}{2}$ and thus $\frac{p+1}{2}=3$ since $\frac{p+1}{2}$ is a prime. Hence $p=5$. In this case, $G \cong \operatorname{PSL}(2,5) \cong A_{5}$, contradicting our assumption. Analogously, $3 \mid p-1$ yields $p=7$. Then $G$ has a maximal subgroup isomorphic to $S_{4}$ which is neither nilpotent nor a Schmidt group. This is a contradiction.

2. $G \cong \operatorname{PSL}\left(2,2^{q}\right)$, where $q$ is a prime.

With similar arguments to those used above, we have that $3 \mid 2^{q}-1$ and $q=2$. Then $G \cong \operatorname{PSL}(2,4) \cong A_{5}$, a contradiction.

3. $G \cong \operatorname{PSL}\left(2,3^{q}\right)$, where $q$ is an odd prime.

We again argue as in the above cases. Since $p$ is odd and $\frac{3^{q}+1}{2}, \frac{3^{q}-1}{2}$ are consecutive integers, it implies only one of them is a power of 2 .

Assume first $\frac{3^{q}+1}{2}=2^{\alpha_{1}}$ for some integer $\alpha_{1}$. Then

$$
3^{q}+1=2^{\alpha}
$$

where $\alpha=\alpha_{1}+1$. Write $q=2 k+1$. If $\alpha \geq 3$, we can take classes module 8 to conclude that $\overline{3}^{2 k+1}+\overline{1}=\overline{0}$, and so $\overline{4}=\overline{0}$. This contradiction yields $\alpha=1$ or 2 . As a result, $q=0$ or 1 . This is impossible. 
Hence

$$
3^{q}-1=2^{\beta_{1}}
$$

for some integer $\beta_{1}$. We can argue as above to get a contradiction.

4. $G \cong \operatorname{PSL}(3,3)$.

In this case, $G$ has a subgroup isomorphic to $\operatorname{GL}(2,3)$ which is neither nilpotent nor a Schmidt group.

5. $G \cong \mathrm{Sz}\left(2^{q}\right)$, where $q$ is an odd prime.

It is known that $G$ has a subgroup isomorphic to $\mathrm{Sz}(2)$, which is neither nilpotent nor a Schmidt group.

Consequently, $G$ cannot be a non-abelian simple group, and this contradiction establishes the theorem.

Proof of Theorem D. We use induction on the order of $G$. We may assume that $G$ is not a Rose group by Theorem A. Then, by Lemma 2.5, quotient groups of $G$ which are non-nilpotent also are $N N C$-groups. Let $\mathscr{A}$ be a Schmidt covering of $G$, and let $N$ be a minimal normal subgroup of $G$ contained in $G^{\mathfrak{N}}$. Then $G / N$ is either nilpotent or an $N N C$-group. Therefore the Fitting length of $G / N$ is at most three. If $N \leq \Phi(G)$, then $G$ has length at most three since the class of groups of Fitting length at most three is a saturated formation. Assume that $N \not \leq \Phi(G)$. Let $M$ be a maximal subgroup of $G$ such that $G=N M$. If $M$ does not belong to $\mathscr{A}$, then $M$ is nilpotent and hence the Fitting length of $G$ is two. Suppose that $M \in \mathscr{A}$. Since $G=G^{\mathfrak{N}} M$, we conclude that $M$ is abnormal in $G$. Then $M$ is a Rose group by Proposition 2.4. Applying Theorem A, $M$ has Fitting length at most two, and so the Fitting length of $G$ is at most three. The proof of the theorem is now complete.

Proof of Theorem E. Assume that the result is false, and let the $N N C$-group $G$ provide a counterexample of minimal order. Put $\mathscr{A}=\left\{K_{1}, \ldots, K_{n}\right\}$.

Let $N$ be a minimal normal subgroup of $G$ contained in $G^{\mathfrak{N}}$. Then Lemma 2.5 implies that $G / N$ is either nilpotent or an $N N C$-group.

Assume that $G / N$ is an $N N C$-group. There are two possibilities:

1. There exists $j \in\{1, \ldots, n\}$ such that $\operatorname{Core}_{G}\left(K_{j}\right)=1$. In this case, $G$ is a primitive group and $G=N K_{j}$. Write $A=K_{j}$. Then $A$ is a Rose group and $A^{\mathfrak{N}}$ is a $q$-group for some prime $q \neq p \in \pi(N)$. Moreover, all core-free maximal subgroups of $G$ are conjugate (see $[1,1.1 .10]$ ). Let 
$C$ be a Carter subgroup of $A$. Then $A=A^{\mathfrak{N}} C$ and $N C$ is a proper subgroup of $G$ supplementing the nilpotent residual of $G$.

Assume that $N C$ does not belong to $\mathscr{A}$. Since $\mathscr{A}$ is a Schmidt covering of $G, N C$ is nilpotent. Suppose that we have two distinct primes dividing the order of the Hall $q^{\prime}$-subgroup of $A$. Then there exists a Sylow subgroup of $N C$ centralising $N$. This contradicts the fact that $C_{G}(N)=N([1,1.1 .7])$. This contradiction implies $|\pi(G)| \leq 3 \leq l+2$, against our choice of $G$.

Consequently, we may assume that $N C$ is a maximal subgroup of $G$ belonging to $\mathscr{A}$. All these maximal subgroups are conjugate in $G$ because $C$ is a Carter subgoup of $G$ and all Carter subgroups are conjugate (see $[1,2.3 .2])$. Put $\pi(C)=\left\{p_{1}, \ldots, p_{t}\right\}$. Then $|\pi(G)| \leq t+2$. If $t=1$, then $|\pi(G)| \leq 3 \leq l+2$. Hence we may assume that $t>1$. Let $C_{i}$ be a maximal subgroup of $C$ such that $\left|C: C_{i}\right|=p_{i}$ for all $i \in\{1, \ldots, t\}$. We have that $S_{i}=N A^{\mathfrak{N}} C_{i}$ is a non-nilpotent maximal normal subgroup of $G$ for all $i \in\{1, \ldots, t\}$, and hence $\left\{S_{1}, \ldots, S_{t}\right\}$ is contained in $\mathscr{A}$. This means that $l \geq t+2$. This contradicts our supposition.

2. $\operatorname{Core}_{G}\left(K_{j}\right) \neq 1$ for all $j \in\{1, \ldots, n\}$. Assume there exists $i \in\{1, \ldots, n\}$ such that $C=K_{i}$ is an abnormal maximal subgroup of $G$. Let $L$ be a minimal normal subgroup of $G$ contained in $C$. Then $G / L$ is not nilpotent, and by Lemma $2.5, \mathscr{C}=\left\{K_{j} / L: L \leq K_{j}, K_{j} / L\right.$ is nonnilpotent $\}$ is a Schmidt covering of $G / L$. Assume that $\mathscr{C}$ is empty. Then every maximal subgroup of $G / L$ is nilpotent and so $G / L$ is a Schmidt group. In this case $|\pi(G / L)|=2$ and so $|\pi(G)| \leq 3$, contrary to supposition. Assume that $\mathscr{C}$ is non-empty. If $L$ is not contained in some $K_{t}$ for some $t \in\{1, \ldots, n\}$, then the minimal choice of $G$ forces $2 \leq|\pi(G / L)| \leq(l-1)+2$. Therefore $2 \leq|\pi(G)| \leq l+2$. Hence we can assume that $L$ is contained in every element of $\mathscr{A}$. If $G / L$ were a Rose group, then $C / L$ would be nilpotent and the number of conjugacy classes of maximal subgroups in $\mathscr{C}$ is less or equal than $l-1$. The minimal choice of $G$ would imply $2 \leq|\pi(G / L)| \leq(l-1)+2$ and then $2 \leq|\pi(G)| \leq l+2$. This would be a contradiction. Therefore we may assume that $C / L$ is not nilpotent. Since $C$ is a Rose group by Lemma 2.4, it follows that $L$ is not a Sylow subgroup of $C$. This means that $|\pi(G / L)|=|\pi(G)|$. The minimality of $G$ and Lemma 2.5 lead $2 \leq|\pi(G)| \leq l+2$, contrary to assumption.

Then we may assume that every maximal subgroup of $G$ belonging to $\mathscr{A}$ is normal in $G$ and so $N \leq \cap\left\{K_{i}: 1 \leq i \leq n\right\}$. If $N$ were not a Sylow subgroup of $G$, then theorem would be applied to the the group 
$G / N$, and we would get $2 \leq|\pi(G)| \leq n+2$. Thus we may assume that $N$ is a Sylow subgroup of $G$ and so $N$ is a complement of an abnormal maximal subgroup of $G$. This means that $N=G^{\mathfrak{N}}$, and $G / N$ is nilpotent. This impossibility clearly shows that $G / N$ must not be an $N N C$-group.

If $G / N$ is nilpotent, then $N=G^{\mathfrak{N}}$, and $N \leq \cap\left\{K_{i}: 1 \leq i \leq n\right\}$ because otherwise some of the subgroups in $\mathscr{A}$ would be nilpotent, and $G$ is a Rose group. The minimal choice of $G$ implies that $N$ is a Sylow subgroup of $G$, and so $N$ is complemented by a nilpotent Hall subgroup $C$ of $G$. Write $\pi(C)=\left\{p_{1}, \ldots, p_{t}\right\}$. Again we may assume that $t>2$. Let $C_{i}$ be a maximal subgroup of $C$ such that $\left|C: C_{i}\right|=p_{i}$ for all $i \in\{1, \ldots, t\}$. Then $S_{i}=N C_{i}$ belongs to $\mathscr{A}$ for all $i$. We conclude that $t \leq n$ and so $|\pi(G)|=t+1 \leq n+2$. This final contradiction proves the result.

\section{Acknowledgements}

The first and second author have been supported by the research grant MTM2010-19938-C03-01 from MICINN (Spain). The third author has been supported by the China Postdoctoral Science Foundation funded project (No: 20100480582) and National Natural Science Foundation of China (No: 11101258). The fourth author has been supported by the National Natural Science Foundation of China (No: 11171243).

\section{References}

[1] A. Ballester-Bolinches and L. M. Ezquerro. Classes of Finite Groups, volume 584 of Mathematics and its Applications. Springer, New York, 2006 .

[2] J. C. Beidleman and H. Heineken. Finite minimal non- $T_{1}$-groups. $J$. Algebra, 319(4):1685-1695, 2008.

[3] J. C. Beidleman and H. Heineken. Minimal non- $\mathcal{F}$-groups. Ric. Mat., $58(1): 33-41,2009$.

[4] J. H. Conway, R. T. Curtis, S. P. Norton, R. A. Parker, and R. A. Wilson. Atlas of Finite Groups. Oxford Univ. Press, London, 1985.

[5] B. Huppert. Endliche Gruppen I, volume 134 of Grund. Math. Wiss. Springer Verlag, Berlin, Heidelberg, New York, 1967. 
[6] B. Huppert and N. Blackburn. Finite groups III, volume 243 of Grund. Math. Wiss. Springer-Verlag, Berlin, Heidelberg, New York, 1982.

[7] B. Huppert and W. Lempken. Simple groups of order divisible by at most four primes. Izv. Gomel. Gos. Univ. Im. F. Skoriny, 3:64-75, 2000.

[8] C. H. Li and H. Zhang. The finite primitive groups with soluble stabilizers, and the edge-primitive $s$-arc transitive graphs. Proc. London Math. Soc. (3), 103:441-472, 2011.

[9] Q. Li and X. Guo. On generalization of minimal non-nilpotent groups. Lobachevskii J. Math., 31(3):239-243, 2010.

[10] Q. Li and X. Guo. On p-nilpotence and solubility of groups. Arch. Math. (Basel), 96:1-7, 2011.

[11] Q. Li and X. Guo. On p-nilpotence and solubility of groups. Arch. Math. (Basel), 96:1-7, 2011.

[12] J. S. Rose. The influence on a finite group of its proper abnormal structure. J. London Math. Soc., 40:348-361, 1965.

[13] O. J. Schmidt. Über Gruppen, deren sämtliche Teiler spezielle Gruppen sind. Mat. Sbornik, 31:366-372, 1924.

[14] L. I. Šidov. Maximal subgroups of finite groups. Sibirsk. Mat. Ž., 12:682683, 1971. 